UWS Academic Portal

\title{
A burst and congestion-aware routing metric for RPL protocol in loT network
}

Altwassi, Hussien Saleh; Pervez, Zeeshan; Dahal, Keshav

Published in:

Proceedings of the 2019 13th International Conference on Software, Knowledge, Information Management and Applications (SKIMA)

DOI:

10.1109/SKIMA47702.2019.8982460

Published: 06/02/2020

Document Version

Peer reviewed version

Link to publication on the UWS Academic Portal

Citation for published version (APA):

Altwassi, H. S., Pervez, Z., \& Dahal, K. (2020). A burst and congestion-aware routing metric for RPL protocol in loT network. In Proceedings of the 2019 13th International Conference on Software, Knowledge, Information Management and Applications (SKIMA) (IEEE Proceedings). IEEE.

https://doi.org/10.1109/SKIMA47702.2019.8982460

\section{General rights}

Copyright and moral rights for the publications made accessible in the UWS Academic Portal are retained by the authors and/or other copyright owners and it is a condition of accessing publications that users recognise and abide by the legal requirements associated with these rights.

Take down policy

If you believe that this document breaches copyright please contact pure@uws.ac.uk providing details, and we will remove access to the work immediately and investigate your claim. 
“(C) (C) 2020 IEEE. Personal use of this material is permitted. Permission from IEEE must be obtained for all other uses, in any current or future media, including reprinting/republishing this material for advertising or promotional purposes, creating new collective works, for resale or redistribution to servers or lists, or reuse of any copyrighted component of this work in other works." 


\title{
A Burst and Congestion-Aware Routing Metric for RPL Protocol in IoT Network
}

\author{
Hussien Saleh Altwassi*, Zeeshan Pervez, Keshav Dahal \\ ${ }^{*}$ School of Computing, Engineering and Physical Sciences \\ University of the West of Scotland \\ Paisley, PA1 2BE, UK \\ \{Hussien.Altwassi, Zeeshan.Pervez, Keshav.Dahal\}@uws.ac.uk
}

\begin{abstract}
The packet loss and power consumption are the main issues considered once congestion occurs in any network, such as the Internet of Things (IoT) with a huge number of sensors and applications. Since IPv6 Routing Protocol for Low Power and Lossy Networks (RPL) is not initially designed for high stream traffic load, this restricts the application domain of RPL in several IoT scenarios such as burst traffic scenarios. The performance of RPL suffers in a network with burst traffic load, which leads to reducing the lifetime of the network and causing traffic congestion among the neighbour nodes. Therefore, to address this issue, we proposed a Burst and CongestionAware Metric for RPL called BCA-RPL, which calculates the rank, considering the number of packets. Also, the proposed mechanism includes congestion avoiding and load balancing techniques by switching the best parent selection to avoid the congested area. Our scheme is built and compared to the original RPL routing protocol for low power and lossy network with OFO (OF0-RPL). Simulation results based on Cooja simulator shows BCA-RPL performs better than the original RPL-OF0 routing protocol in terms of packet loss, power consumption and packet delivery ratio (PDR) under burst traffic load. The BCA-RPL significantly improves the network where it decreases the packet loss around $50 \%$ and power consumption to an acceptable level with an improvement on the PDR of the IoT network.
\end{abstract}

Index Terms-Internet-of-Things, RPL, Routing, Objective Function, Packet Loss, Load Balancing, and Congestion Avoiding.

\section{INTRODUCTION}

In routing protocol for low power and lossy networks (RPL) load balancing is managed by using a route request technique which selects the efficient path without dealing with the congested area. However, in a burst traffic scenario, multiple sensor nodes in a network start generating high data packet rate which causes congestion. Thus, packet overflow at buffer nodes occurs in the network [1].

The performance of RPL on evaluation and analysis basis has been studied intensively in the literature with varying scenarios and network setups. In the study [2] the authors perform an extensive experiment in order to analyze the performance of the RPL routing protocol in terms of topology stability. Similarly, the authors in [3] evaluate the RPL performance using the data collection model, the author compares the results with the Collection Tree Protocol (CTP), the comparison shows that RPL has better performance in terms of Packet Reception Ratio (PRR) and power consumption. The study in [4] investigates the two standard RPL objective functions using different density networks, in terms of PDR and power consumption, where each node is placed under random and grid topology. Through different experimentations, the authors demonstrate the packet reception ratio affected the performance of the routing structure. Similarly, the authors in [5] survey the RPL with exhaustive information on building RPL routing protocol, objective function, DODAG models, and the best route selection for nodes in Low Power and Lossy Networks (LLNs).

Since traffic congestion causes remarkable issues for IoT networks, supporting a burst traffic network with a load balancing feature can help to mitigate the impact. We can leverage IoT to drive venue efficiency in burst traffic network through several initiatives such as traffic management, building performance, and stadium security to improve the game-day experience as shown in Fig.1. In our study, we monitor all building systems from a centralized area to track metrics such as energy costs and optimize building performance using different network density. This will help people enter and exit the venue, find parking, and navigate to various parts of the stadium by using mobile apps, cameras, and sensors especially in the peak time when congestion occurs.

In this study, through simulation of bust traffic scenario, we demonstrate high packet loss and power consumption with the standard RPL with OF0. We introduced an effective improvement to support RPL performance in the burst traffic scenarios even in the high-density network. We focused on reducing the packet loss as well as the power consumption within a good PDR in LLN, we used two techniques, avoiding congestion technique by switching the parent node, and load balancing technique by considering the number of packets in the parent selection algorithm.

The rest of the paper is organized as follow. Section II mentions the background and related work. Section III defines the problem with RPL protocol for burst traffic. In Section IV, we present our solution. Section V shows performance evolution and enhancement. In Section VI, we conclude our 


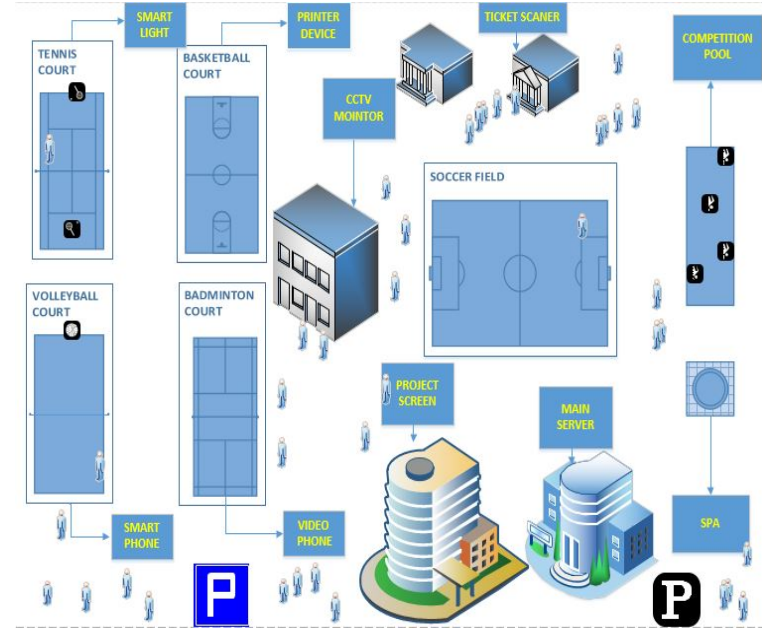

Figure 1. Architecture of stadium and sport event automation control system.

work.

\section{BACKGROUND AND RELATED WORK}

The authors in [6] have recommended two groups of RPL routing metric set which are node routing and link metrics. Also, they have set a constraint. The routing metrics and constraints are proposed to fit the needs of LLNs. For example, node metric can be hop count, node energy, node state or a combination set of the node metrics. Also, Expected Transmission Unit (ETX), latency, and throughput as an example of the link metrics which could be combined as a composite metric (more than one metric is merged together). The authors in [7] have implemented the ETX and the HOP Count (HC) for path selection in order to assess the performance of RPL in terms of control traffic overhead, PDR, power consumption and network latency. The simulator results demonstrate that selecting a path with ETX metric in the non-dense network has better performance than the selected via HC metric. However, few papers have been presented to investigate a new RPL objective function in LLNs networks [8], [9], [10], [3], [11], [12] and [13], however still there is an absence of studies about load balancing and congestion problem in RPL over burst traffic in LLNs. We review previous studies in two categories: (1) load balancing protocols, (2) congestion-aware protocol.

\section{A. Load Balancing Protocols}

In the study [8], a new load balancing mechanism was optimized called LB-RPL, it amplified the queue utilization to allow a node to arrange its parent candidates. However, when the congestion occurred, a delay was noticed for distributing the routing information which was collected from the base of the neighbour nodes.

The authors in [9] showed their interests to solve the load balancing issue under heavy traffic scenarios. They merged the standard objective function zero and queue information to support RPL routing to be balanced under heavy data traffic. The main restriction on their study was the use of OF0, which is not suitable for large density and dynamic IoT networks. We mention that QU-RPL has the advantage of improving queue losses and PDR. Although, QU-RPL does not support the hybrid network as well as it increases the control overhead packet.

\section{B. Congestion-Aware Protocol}

The authors in [10] tackled the congestion problem and proposed the congestion-aware objective function called CAOF to deal with high data transmission. The buffer occupancy was the main factor in specifying the efficient route in this study. This work focused on avoiding the congestion area, to allow the node to choose the efficient routing where the PDR was the target. On the other hand, the attention for routing stability had not been covered. The study in [14] handled the congestion problem using parent selection mechanism by employing the queue utilization and the residual energy metric of neighbouring nodes. The contribution of this study to provide an effective selecting mechanism for the proper parent node which will reduce the queue congestion and more residual energy than others. The experiments of the network simulator were carried out using the Cooja simulator under random and grid topology. The results showed a superior routing performance in terms of and PDR and power consumption.

The authors in [3] approached another congestion solution called M-RPL which delivered two preferred parent nodes when neighbouring nodes distributed the traffic towards the parent using the control messages of RPL. Therefore, a mechanism with more than one preferred parent will increase the chances to avoid congestion. The authors in [11] provided a new routing metric to discover bottlenecks each path toward the root. They used parent rank as well as ETX for rank computation. While the authors in [15] presented a mechanism to provide a stable network. The new mechanism estimated the amount of traffic on a node by taking the traffic as a main factor in the routing process. However, the mechanism was feasible for all traffic conditions because it worked with bidirectional and symmetric links only.

From the above limitations, these previous contributions do not provide an efficient route which handles load balancing and the congestion problem in burst traffic scenarios. Moreover, the response to these problems in terms of routing information update is very slow. Moreover, the main issue where the frequent parent switching is carried out is the overhead on the network and to solve this problem, we are proposing a load balancing and congestion-aware metric under burst traffic scenarios.

\section{Problem with RPL Protocol for Burst Traffic}

RPL fails to distribute traffic load with the best path while a burst mode applied, it is regarding the missing of the load balancing features [16]. In fact, RPL is classified as a single path routing protocol and in this case, the nodes will transmit packets directly to the preferred parent [17]. Unfortunately, in burst traffic scenarios, the networks need to choose links to build a new topology regarding the number of times that a set 
of nodes will switch their prefer parents repeatedly in order to address the load balancing issue. This will cause congestion and unnecessary cost. Therefore, we propose a congestionaware routing metric that is a composite of three metrics including OF0, rank, and the number of received packets.

The RPL routing protocol was designed for handling the low power and lossy network, there are several features still missing and need to be covered such as self-healing mechanism as well as load balancing. Regarding this fact, RPL is not able show the capability to support a network with burst traffic, which mean, once the traffic rate becomes in a high level during burst mode, then RPL will not be able to handle it as it should be, comparing to the regular traffic. Regarding this issue, the IoT network with RPL routing protocol will suffer from many problems consist of load imbalance and congestion, where a high packet loss and high-power consumption will be generated. The problem becomes complicated in burst traffic when the network density is increased.

Fig. 2 presents an example of congestion avoiding technique, when a network is suffering from the instability, which is caused once nodes start switching their preferred parent without any methods to check the traffic load in advance to reach load balancing. In the first RPL tree, seven nodes have been selected to build the network, where only one node is a source node $(\mathrm{S})$ and six other nodes are destination nodes. Four nodes from the six nodes have selected node number 1 , with a smaller number of packets when it received DIO from it. However, in the second RPL tree, a new DIO from node 1 with updated routing table information which causes all the four nodes to change the preferred parent to node number 6 , which has a smaller number of packets than node 1 . In fact, it becomes a loop in a switching parent whenever the neighbour's nodes receiving new DIO which ensuing load alternation but with no balancing.

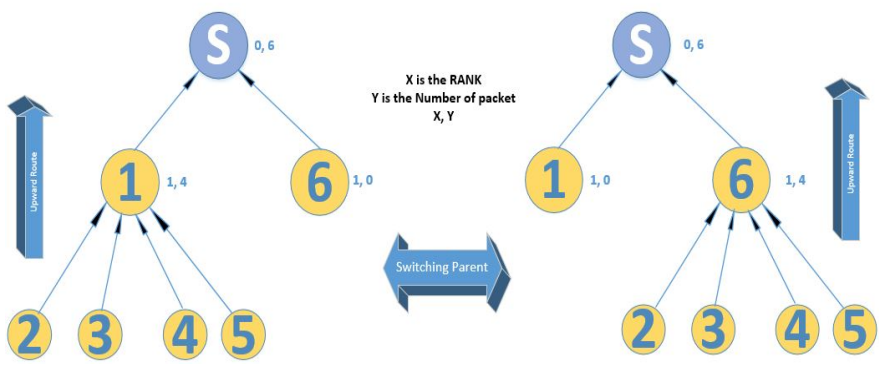

Figure 2. An example of the congestion avoiding technique

\section{Proposed SOLUTION}

In this study, we have solved the load balancing and congestion problem in the RPL routing protocol under burst traffic networks. We introduced a new lexically metric called BCARPL (Load Balancing and Congestion Aware for RPL Routing Protocol). The proposed metric applies a load balancing using the hop-count metric as a primary metric to compute the rank. Also, to provide a parent selection mechanism for each node to select it neighbour's node as candidate parent; whenever the candidate parent set is selected, the second metric focus on switching to another parent with respect to the number of packets received from neighbours in order to avoid the congestion area.

\section{A. The Load-Balancing Technique}

In the RPL routing process, nodes are sending a packet to another node including routing information in order to build the RPL DODAG [18]. The routing information includes ID node, queue size, received packet, and power, among others. Also, each node receives a specific number of packets from the neighbour nodes during this procedure is part of the load balancing technique [19]. To do so, we monitor the number of packets in order to assess the traffic load as an important factor in choosing an efficient route. Also, we provide a proper arrangement to compute the path with exact intervals. In this case, we will build the path every time based on the present value of the traffic load for the nodes with less power consumption, each time the node is sending a packet. In fact, we need an efficient route to control the traffic load whenever the number of packets on a path increases. Thus, the link efficiency of the initial optimal path needs to be adjusted with the traffic load information on the path. Therefore, we provide a technique to compute the path dynamically on exact time based on the number of packets. The traffic size of each node becomes balanced via the network by distributing the traffic across all the neighbour nodes with a smaller number of packets.

\section{B. The Congestion Avoiding Technique and Parent Selection Algorithm}

Our scheme avoids the bottlenecks area in a burst traffic scenario. The proposed OF lexically combines the hop count with the traffic indicator metric (TraffickInd). The hop count metric is employed by a node to select candidate parents and then the traffic indicator metric is used to apply the load balancing technique in case of having more than one candidate parent in the parent set. Fig. 3 showed a flow chart of the BCARPL implementation over RPL. In this section, we describe the implementation of load balancing technique and merge it with the congestion avoiding of the high packet stream nodes over the standard RPL routing protocol using the OF0.

The steps of our proposed algorithm for the parent selection procedure are shown in Algorithm 1. The traffickInd metric is calculated by each node in order to consider the number of received packets from its children over a specified period of time. In order to implement BCA-RPL, several enhancements have been made to the standard RPL routing protocol. These enhancements are mentioned as steps in the following:

Stage 1: whenever any node in the network sends a message to another node, the sender node must check the information of its routing table in order to find a route towards the destination node. In this case, if the path is available in the routing table then $\mathrm{S}$ will send the message to the neighboured node. If it is not available in the routing table then the message stays in a 


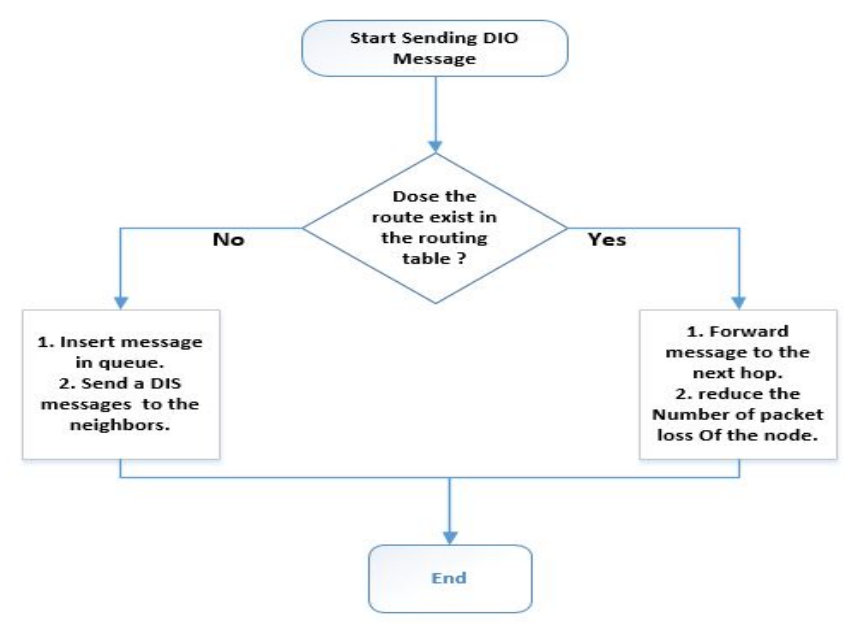

Figure 3. Flow Chart of the new technique over the RPL.

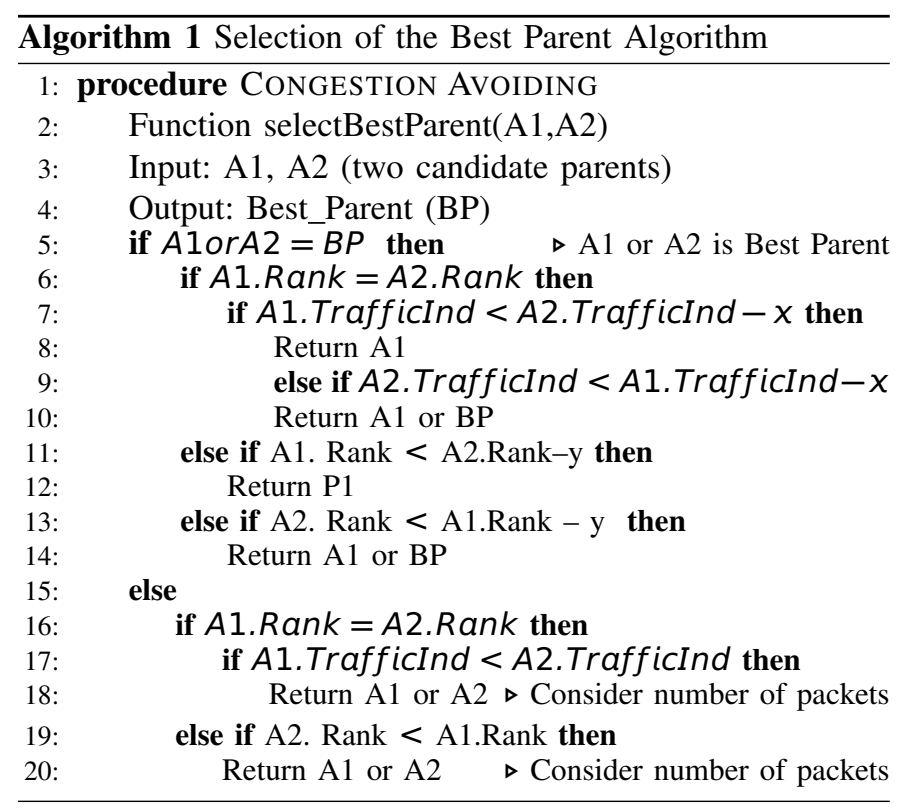

queue. Also, the $\mathrm{S}$ node starts to send DIS to its neighbours to initiate the discovery process.

Stage 2: we have reduced the number of packets loss of the node that involved in building the RPL DODAG tree based on the congestion avoiding technique. However, the PDR of these nodes should be enough to send the data packet over burst traffic to reach the following neighbour in the path.

Stage 3: to merge the load balancing technique with the congestion avoidance technique, we apply a method before sending the messages to the following hop. This process checks the number of packets of the sender node. If the number of packets in the node is relatively high, then the process will turn into another candidate node in order to avoid the congestion. After doing this, the current path deleted from the routing table. This leads the $\mathrm{S}$ node to initiate the discovery process again using the DIO messages and finds a new path to the node in order to achieve load balancing.
Table I

SIMULATION PARAMETERS.

\begin{tabular}{ll}
\hline Parameters & Values \\
\hline Objective Function (OF) & OF0-RPL, BCA-RPL \\
Traffic Load (PPS) & 30pps \\
Density Network & $10,20,30,40$ AND 50 Motes \\
TX Ratio & 1 \\
RX Range & 100 \\
Mote Startup Delays & 1 \\
Topologies & Random \\
Simulation Time & 900 second \\
\hline
\end{tabular}

\section{Performance Evaluation}

In this study, we evaluated the RPL routing protocol and optimize it in terms of packet loss, power consumption and PDR. RPL performance is analyzed after extensive experimentation using Cooja simulator on Contiki operation system. The simulator results showed that the congestion and unbalanced traffic are increased in the burst traffic scenarios, especially when the high-density network is increased.

\section{A. Simulation Environment Setup}

We have used the standard IoT simulator, called Cooja simulator, it is been known as a very suitable tool for IoT network development. To run our scenario, we have used the SKY mote type to build a network with one source node (sink) located in the point of $(50,50)$ and the remaining destination nodes (sender) are distributed randomly, all the nodes are distributed within 1000 meters in a squared area. As in Table I, we have employed two metrics in our RPL network including OF0-RPL and BCA-RPL in order to compare the standard OF-RPL with the enhanced BCA-RPL. Also, the network consists of different density of nodes $(10,20,30,40,50)$ the experiments have been verified in a burst traffic scenario with 30 apps.

\section{B. Results}

The simulation results were compiled for experiments under burst traffic load networks using random typologies. We evaluated the performance of RPL using OF0-RPL and BCARPL. We located the sink in the middle. The results present the impact of the used metric on the RPL behaviour in terms of packet loss rate, power consumption, and PDR as shown in Fig. 4. Congestion increases as the density network increases where the problems of packet loss and power consumption will get worse. Therefore, it becomes more complicated to reduces the traffic load balancing opportunities in the network.

- The RPL packet loss rate within OF0-RPL and BCA-RPL

In Fig. 5, we observed that the packet loss rate increased in a regular manner when the number of nodes increased for both OF0-RPL and BCA-RPL, except for the network with 10 to 20 nodes where the packet loss ratio is zero even in burst traffic. Also, suddenly the packet loss ratio value increases once the density network is 30 nodes under burst traffic (30 or more). Moreover, a high packet loss is recorded once RPL-OF0 is used in a high-density network with burst traffic scenarios and 


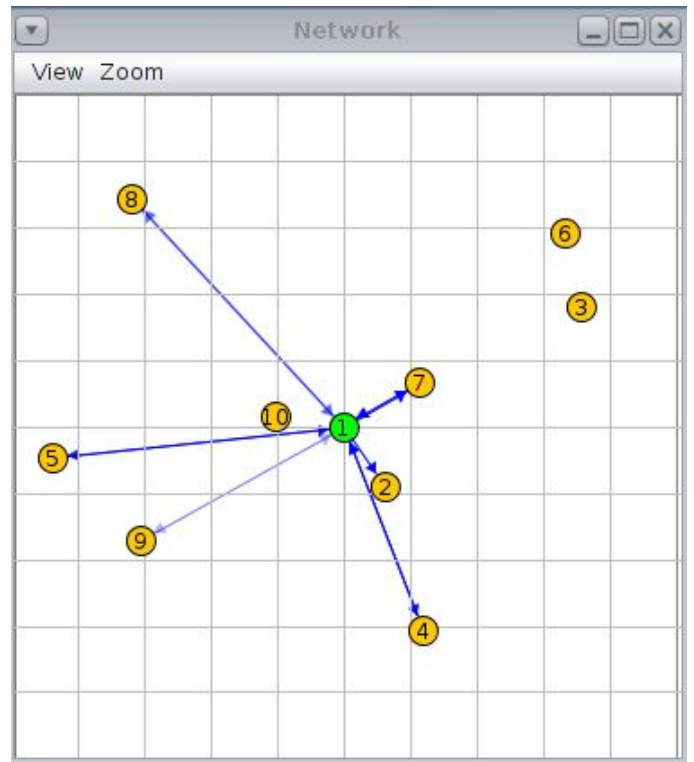

(a) 10 Nodes

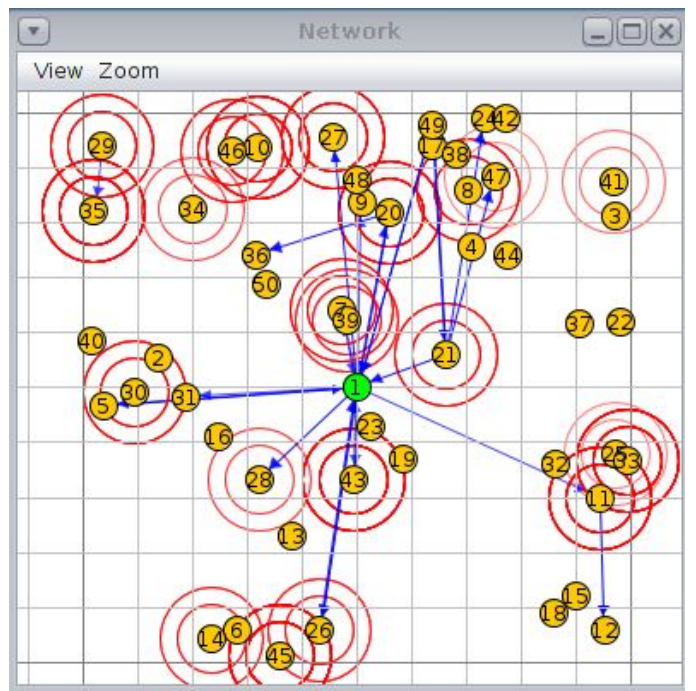

(b) 50 Nodes

Figure 4. Network simulator window with 10 nodes and 50 nodes.

reached to $74 \%$ and $91 \%$ for (40 and 50 nodes respectively). While these percentages are reduced for the same scenarios to become only $39 \%$ and $46 \%$ for (40 and 50 nodes respectively) using our new metric BCA-RPL.

- The RPL power consumption impact within OF0-RPL and BCA-RPL

Fig. 6, demonstrates the power consumption RPL with BCA-RPL is better than the OF0-RPL - the power consumption increases as network density increases. Also, the results show that the power consumption values of OF0-RPL in a lowdensity network (10 to 20 nodes) is about $2.75 \mathrm{~kb}$ to $2.89 \mathrm{~kb}$ respectively. While it is 2.83 to 2.94 once BCA-RPL is used than 0.98 whenever the burst traffic mode is generated (more than $30 \mathrm{pps}$ ). Moreover, we observed that the highest values

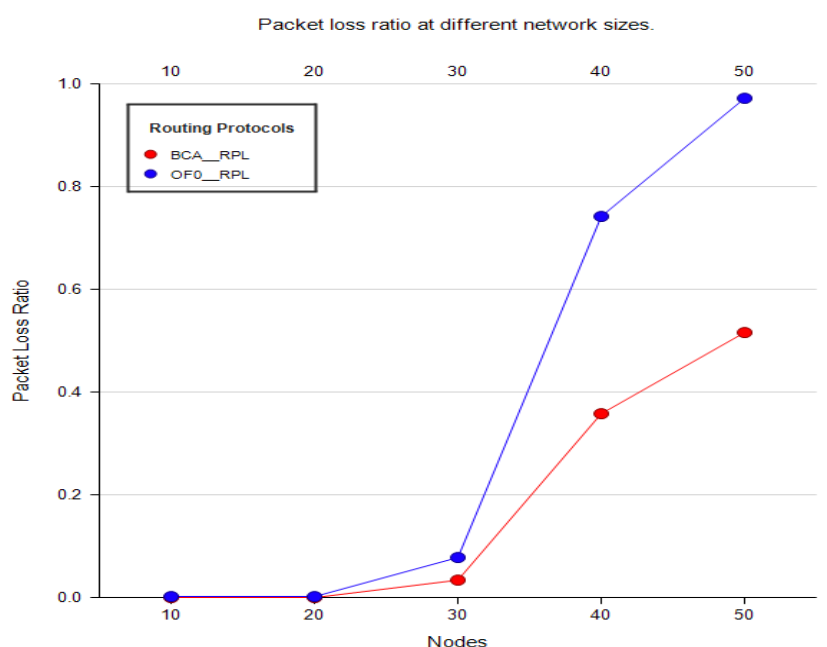

Figure 5. The packet loss ratio values under different network density.

of power consumption occur once a network with the highdensity network of 50 nodes is applied for both OF0-RPL and BCA-RPL, where power consumption values reached $7.32 \mathrm{~kb}$ for OF0-RPL and $6.08 \mathrm{~kb}$ for BCA-RPL. It means BCA-RPL is reducing the usage of the power of RPL routing protocol under burst traffic in different density network especially in the high-density network.

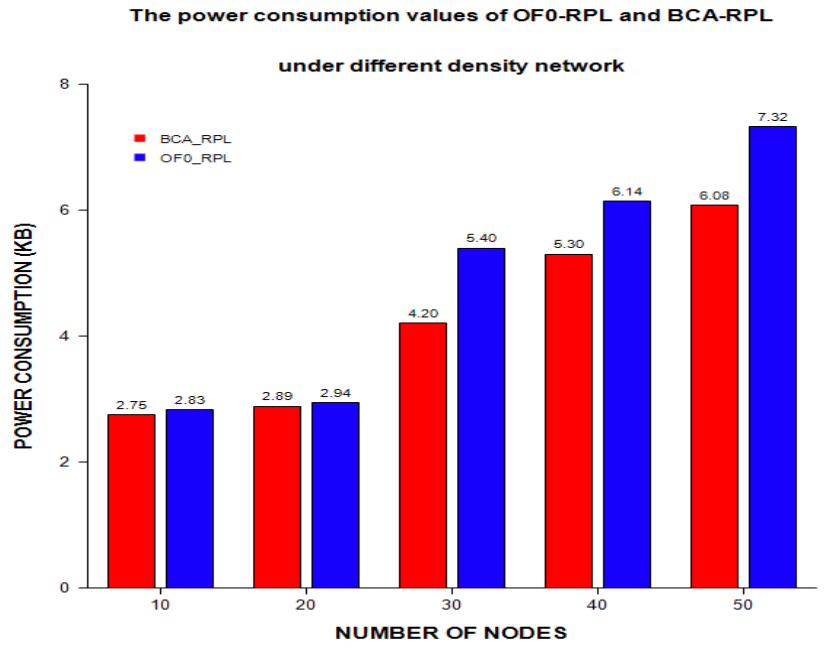

Figure 6. The power consumption values under different network density.

- The RPL packet delivery ratio impact within OF0-RPL and BCA-RPL

Fig. 7 demonstrates the BCA-RPL enhances PDR for OF0RPL with almost $1.00 \%$ PDR for both metrics OF0-RPL and BCA-RPL once 10, 20 and 30 nodes are used under burst traffic. On the other hand, the PDR is suddenly decreased once the number of nodes reaches to 40 nodes in both metrics OF0-RPL and BCA-RPL with $0.62 \%$ and $0.64 \%$ respectively. 
Similarly, once the number of nodes is increased to 50 nodes, a lowest PDR percentage was recorded with 0.48 and 0.54 for OF0-RPL and BCA-RPL respectively.

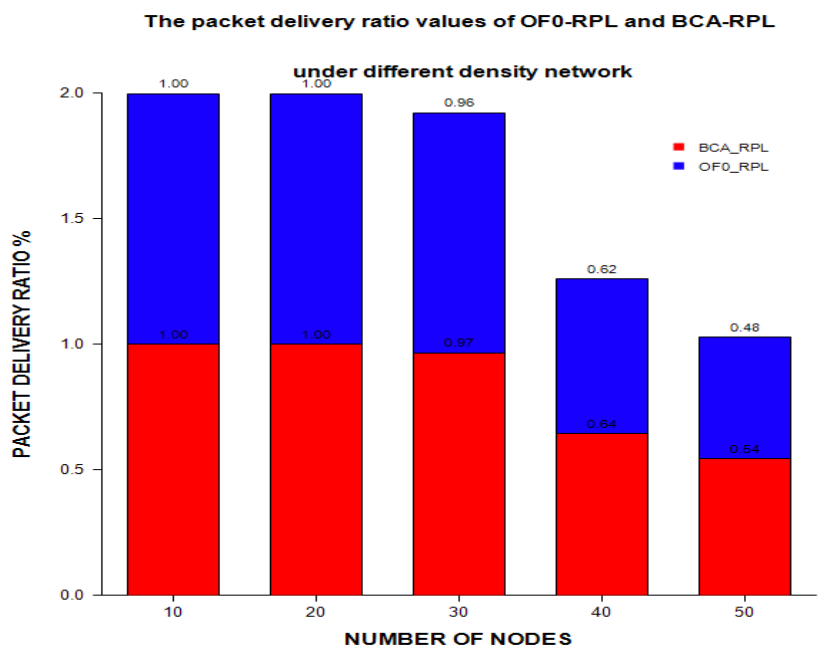

Figure 7. The PDR values under different network density.

\section{CONCLUSION}

In this work, we have tackled two major issues with RPL: the load balancing and congestion area in the RPL routing protocol under burst traffic networks. To this conclusion, we introduced a new lexically metric called BCA-RPL. The proposed metric applies two techniques, the first technique is the load balancing technique by using the hop-count metric as a primary metric to compute the rank. The second technique is the congestion avoidance technique by switching the best parent selection to avoid the congested area. By extensive experimentation based on Cooja simulator, we demonstrated that BCA-RPL worked efficiently with RPL and attained an enhancement in terms network packet loss with $50 \%$ reduction, power consumption and PDR with an acceptable level of the IoT network. We compared BCA-RPL and OF0-RPL performance, BCA-RPL performances better than the original OF0RPL under burst traffic load with different density networks.

\section{REFERENCES}

[1] A. Ghaffari, "Congestion control mechanisms in wireless sensor networks: A survey," Journal of Network and Computer Applications, vol. 52, pp. 101 - 115, 2015. [Online]. Available: http://www.sciencedirect.com/science/article/pii/S1084804515000557

[2] O. Gaddour and A. Koubâa, "Rpl in a nutshell: A survey," Computer Networks, vol. 56, no. 14, pp. 3163 - 3178, 2012. [Online]. Available: http://www.sciencedirect.com/science/article/pii/S1389128612002423

[3] M. Ha, K. Kwon, D. Kim, and P.-Y. Kong, "Dynamic and distributed load balancing scheme in multi-gateway based 6lowpan," 092014.

[4] M. Qasem, H. Altawssi, M. B. Yassien, and A. Al-Dubai, "Performance evaluation of rpl objective functions," in 2015 IEEE International Conference on Computer and Information Technology; Ubiquitous Computing and Communications; Dependable, Autonomic and Secure Computing; Pervasive Intelligence and Computing, Oct 2015, pp. 16061613.

[5] E. Aljarrah, M. Bani Yassein, and S. Aljawarneh, "Routing protocol of low-power and lossy network: Survey and open issues," 092016.
[6] C. Systems, M. Kim, K. Pister, N. Dejean, and D. Barthel, "Routing metrics used for path calculation in low-power and lossy networks," 2012.

[7] S. A. Abdel Hakeem, A. A. Hady, and H. Kim, "Rpl routing protocol performance in smart grid applications based wireless sensors: Experimental and simulated analysis," Electronics, vol. 8, no. 2, 2019. [Online]. Available: http://www.mdpi.com/2079-9292/8/2/186

[8] Xinxin Liu, Jianlin Guo, G. Bhatti, P. Orlik, and K. Parsons, "Load balanced routing for low power and lossy networks," in 2013 IEEE Wireless Communications and Networking Conference (WCNC), April 2013, pp. 2238-2243.

[9] H. Kim, H. Kim, J. Paek, and S. Bahk, "Load balancing under heavy traffic in rpl routing protocol for low power and lossy networks," IEEE Transactions on Mobile Computing, vol. 16, no. 4, pp. 964-979, April 2017.

[10] H. A. A. Al-Kashoash, M. Hafeez, and A. H. Kemp, "Congestion control for 6lowpan networks: A game theoretic framework," IEEE Internet of Things Journal, vol. 4, no. 3, pp. 760-771, June 2017.

[11] Q.-D. Nguyen, J. Montavont, N. Montavont, and T. Noël, "Rpl border router redundancy in the internet of things," in Ad-hoc, Mobile, and Wireless Networks, N. Mitton, V. Loscri, and A. Mouradian, Eds. Cham: Springer International Publishing, 2016, pp. 202-214.

[12] S. Sarwar, S. Rauf, R. Rasheed, and L. Aslam, "Energy-aware routing in internet of things (iot)," in 2019 2nd International Conference on Communication, Computing and Digital systems (C-CODE), March 2019, pp. 81-86.

[13] L. Nguyen and C. Kim, "Towards skewness and balancing of RPL trees for the internet of things," CoRR, vol. abs/1903.01839, 2019. [Online]. Available: http://arxiv.org/abs/1903.01839

[14] R. Ullah, Y. Faheem, and B. Kim, "Energy and congestion-aware routing metric for smart grid ami networks in smart city," IEEE Access, vol. 5, pp. 13799-13 810, 2017.

[15] M. Michel, S. Duquennoy, B. Quoitin, and T. Voigt, "Load-balanced data collection through opportunistic routing," in 2015 International Conference on Distributed Computing in Sensor Systems, June 2015, pp. 62-70.

[16] H. Altwassi, Z. Pervez, K. Dahal, and B. Ghaleb, "The rpl load balancing in iot network with burst traffic scenarios," in 2018 12th International Conference on Software, Knowledge, Information Management Applications (SKIMA), ser. IEEE Conference Proceedings. United States: IEEE, 22019.

[17] P. Thubert, "Objective Function Zero for the Routing Protocol for Low-Power and Lossy Networks (RPL)," RFC 6552, Mar. 2012. [Online]. Available: https://rfc-editor.org/rfc/rfc6552.txt

[18] A. Y. Barnawi, G. A. Mohsen, and E. Q. Shahra, "Performance analysis of rpl protocol for data gathering applications in wireless sensor networks," Procedia Computer Science, vol. 151, pp. 185-193, 2019.

[19] B. Ghaleb, A. Al-Dubai, E. Ekonomou, W. Gharibi, L. Mackenzie, and M. B. Khalaf, "A new load-balancing aware objective function for rpl?s iot networks," June 2018. [Online]. Available: http://eprints.gla.ac.uk/169318/ 